\title{
A Simple Internal Resistance Estimation Method Based on Open Circuit Voltage Test Under Different Temperature Conditions
}

\author{
Qi Yao \\ School of Electrical and \\ Data Engineering \\ University of Technology Sydney \\ NSW, Australia 2007 \\ Email: qi.yao-3@student.uts.edu.au
}

\author{
Dylan Dah-Chuan Lu \\ School of Electrical and \\ Data Engineering \\ University of Technology Sydney \\ NSW, Australia 2007 \\ Email: Dylan.lu@uts.edu.au
}

\author{
Gang Lei \\ School of Electrical and \\ Data Engineering \\ University of Technology Sydney \\ NSW, Australia 2007 \\ Email: Gang.Lei@uts.edu.au
}

\begin{abstract}
State-of-charge (SoC) is one critical parameter for battery management system. SoC cannot be directly measured but it can be estimated according to some information of battery management system such as voltage and current. Two commonly used methods to estimate the SoC are 1) by using current times a constant internal resistance, and 2) by referring to a SoCresistance lookup table to interface with an open-circuit-voltage (OCV)-SoC lookup table. However, these widely used testing methods of internal resistance have not considered the influence of SoC, temperature and current rate. which are in fact related to internal resistance. Therefore, ignoring the temperature and current rate factors will obtain inaccurate internal resistance measurement and battery SoC estimation. This paper hence proposes a dynamic resistance model with improved accuracy through combining SoC-OCV at different ambient temperatures with different discharging rates defined at the standard ambient temperature ( 25 degree) condition. The proposed method will not only improve the accuracy but also reduce the testing time.

Index Terms-Internal resistance, dual-aim, less time, dynamic resistance.
\end{abstract}

\section{INTRODUCTION}

For lithium batteries, the internal resistance model is the most straightforward circuit parameter to characterize the batterys dynamics [1]. There are some advanced models with several parameters such as the equivalent circuit model with one or more resistance-capacitance pairs [2]. They have demonstrated good performance in the experimental environment. However, they might risk over-fitting and increase the complexity of online computation in practical condition [1].

There are different methods to get the direct current internal resistance [1], [3]. The widely used method is short pulse testing method such as hybrid power pulse characterization (HPPC), which uses current pulse to discharge batteries and assume the SoC and temperature will not change at this discharging period [3]. Before each current pulse, the battery will be set for an hour and the measured terminal voltage will be assumed as open circuit voltage. Therefore, the internal resistance at different $\mathrm{SoC}$ has been tested and a $\mathrm{R}$-SoC table can be generated. Nevertheless, this method has some drawbacks. Firstly, in order to make sure the testing is under the same environment, the values of charging current and temperature should be constant for one complete cycle (SoC:1$0)$. It will take a long time to test the battery under different current and temperature conditions. Secondly, this method has been assumed that the $\mathrm{SoC}$ will not change during pulse discharging. But when the battery is in the low voltage region, the voltage is quite sensitive to the variation of SoC. Therefore, this assumption will make the calculation inaccurate.

The second method is called long-pulse-current test. In [4] the battery has been discharged with $3 / \mathrm{C}$ at every $5 \%$ SoC increment. The resistance can be calculated by using voltage drop and current. The paper considered the temperature and SoC but ignored the influence of current rate on resistance.

The third method is data fitting [1], [5]. After getting the SoC-OCV result, the battery would be tested under dynamic current conditions. The parameter $\mathrm{R}$ can be fitted using a sequence of current, voltage and the SoC-OCV table by the least square algorithm. This method gives fast estimation because SoC-OCV and dynamic street testing are fundamental tests for battery modelling and SoC estimation. This method serves two purposes simultaneously without spending extra time on resistance measurement. The disadvantage of this method is that the fitting resistance is a perfect match only under a specific tested current profile. Moreover, this method still has not considered the influence of temperature.

The forth method is called constant current test [6], which uses different constant current values at the same ambient temperature environment to discharge the battery. The paper has considered the influence of different current rates and temperatures, but it ignored the temperature influence on the OCV.

The idea of temperature-current based internal resistance battery model is proposed in this paper. This paper mainly focuses on the resistance measurement method, which is a compromise between accuracy and testing time. SoC-OCV tests at six ambient temperature conditions, and currentterminal voltage test in four different discharging current rates at standard ambient temperature $\left(25^{\circ} \mathrm{C}\right)$ will be discussed in 
TABLE I

BATTERY INFORMATION

\begin{tabular}{lllll}
\hline Type & $\begin{array}{l}\text { Nominal } \\
\text { Voltage }\end{array}$ & $\begin{array}{l}\text { Nominal } \\
\text { Capacity }\end{array}$ & $\begin{array}{l}\text { Upper/Lower } \\
\text { Voltage }\end{array}$ & $\begin{array}{l}\text { Max Continuous } \\
\text { discharge } \\
\text { current }\end{array}$ \\
\hline LiFePO4 & $3.3 \mathrm{~V}$ & $1.1 \mathrm{Ah}$ & $3.6 / 2.0 \mathrm{~V}$ & $30 \mathrm{~A}$ \\
\hline
\end{tabular}

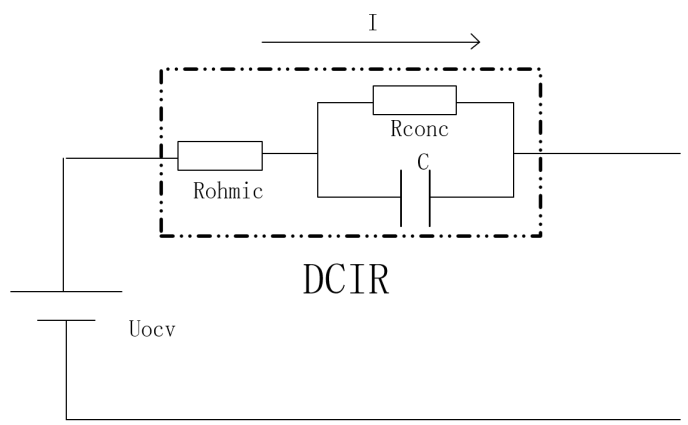

Fig. 1. Internal Resistance Model.

Section II. The data of dynamic stress test (DTS) at seven ambient temperature conditions will be used to fit circuit the internal resistance, and the results will be shown in Section IV.

\section{EXPERIMENT INVESTIGATION}

\section{A. Battery cells under test}

These separate tests were used for model identification, SoC-OCV-T configuration and data analysis, respectively. The tests presented in this paper were performed on A123 (LiFePO4) cells (the key specifications are shown in Table I). To reduce the testing time, part of the results come from University of Maryland battery testing center [1], [7]. This center publishes the testing data in an open source manner. To obtain the desired information about the relationship between different discharging rates and internal resistance values, the battery cells were discharged with different current rates at by our lab.

\section{B. Model Identification}

Equivalent circuit models are widely used method in battery modelling because of their simple structure. A typical model has been shown in Fig. 1. The battery terminal voltage is represented by open circuit voltage $V_{\text {ocv }}$, instantons voltage drop resistance $R_{\text {ohmic }}$ and a transient voltage representing by one RC pair. But as described in Section I, complicated model can provide an accurate result but will require complicated online computation. Therefore, we choose to use an internal resistance (DCIR) to replace these components. This is the easiest method to estimate the SoC because two parameters have been measured online and the value of direct current internal resistance (DCIR) has been calculated before. In this paper, direct current internal resistance (DCIR) will be represented by $\mathrm{R}$ as shown in (1). Then, the SoC could be estimated using the lookup table of SoC-OCV

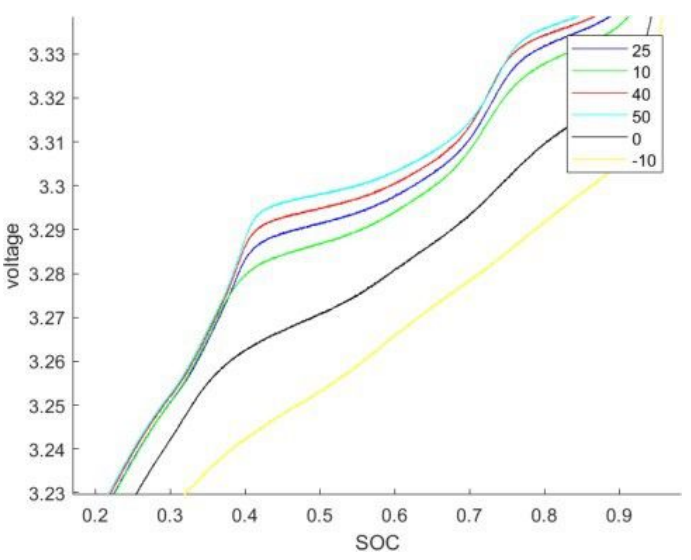

Fig. 2. OCV-SoC curves at different temperature conditions.

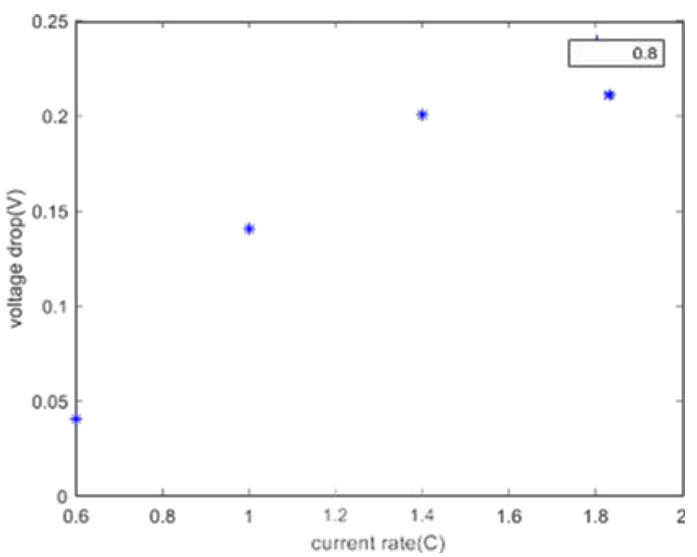

Fig. 3. OCV-Uterm drop at different discharging rates $(\mathrm{SoC}=0.8)$.

$$
\begin{gathered}
U_{\text {term,time }}=U_{\text {ocv }}-I_{\text {time }} \cdot R \\
U_{o c v} \propto f(S O C)
\end{gathered}
$$

\section{OCV-SoC-Temperature Configuration}

In order to get the SoC-OCV-T relationship, six $\mathrm{C} / 20$ discharging tests have been conducted at different ambient temperature values $\left(-10^{\circ} \mathrm{C}, 0^{\circ} \mathrm{C}, 10^{\circ} \mathrm{C}, 25^{\circ} \mathrm{C}, 40^{\circ} \mathrm{C}, 50^{\circ} \mathrm{C}\right)$. As shown in Fig. 2, it can be observed that the $\mathrm{SoC}$ at $0^{\circ} \mathrm{C}$ and $-10^{\circ} \mathrm{C}$ are higher than other temperature conditions when the OCV is at the same value. Therefore, (1) should be updated to $(3)$ :

$$
U_{\text {term,time }}(S o c, T)=U_{o c v}(S o C, T)-I_{\text {time }} \cdot R(T)
$$

\section{Different $C$-rates}

In order to get the R-Current relationship, four discharging tests have been conducted at the Tamb $=25^{\circ} \mathrm{C}(0.6 \mathrm{C}$, $1.0 \mathrm{C}, 1.4 \mathrm{C}$, and $1.8 \mathrm{C}$ ). Fig. 3 shows the voltage drop ( at different discharge rates at the same SoC value. It can be found 
that the relationship between the current and the voltage drop is nonlinear. Hence (1) should be updated to (4):

$$
U_{\text {term,time }}(S o C, I)=U_{o c v}(S o C)-I_{\text {time }} \cdot R(I, S o C)
$$

\section{MORE ACCURATE MODELS}

Based on the analysis of all these coupling factors of a battery model as shown in Section II, it becomes clear that the internal resistance is a function of current rate, temperature and SoC. Traditional pulse test fails to extract these coupling values because the SoC changes when the battery cell has been charged or discharged. This new method, however, can solve this problem through an update of the change of SoC in the estimation calculation. Based on (3) and (4), equation (5) shows the method of internal resistance.

$$
R(I, T, S o C)=\frac{U_{o c v}(S o C, T)-U_{\text {term }, \text { time }}(S o C, T, I)}{I_{\text {time }}}
$$

\section{A. Current-based resistance}

As mentioned in Section II, the battery cell has been tested under different current rates under the same ambient temperature to get the R-current relationship curve. When the SoC and temperature keep at a constant value, the resistance can be calculated by using (6). Since this is not a linear curve, we choose to use an averaging and separating linear region method as shown in (7). Ri2 and Ri1 have been calculated by (4), $I_{\text {new }}$ is a current that has not been conducted, $R_{\text {new }}$ is the resistance at Inew under the same temperature condition with $R_{i 2}$ and $R_{i 1}$.

$$
\begin{gathered}
\frac{\partial U_{\text {term,time }(I)}}{\partial I}=U_{o c v(S o C, T)}-\frac{\partial R(I)}{\partial I} \\
R_{\text {new }}=\left(\frac{R_{i 2}-R_{i 1}}{I_{2}-I_{1}}\right) \cdot I_{\text {new }}+R_{i 1}
\end{gathered}
$$

\section{B. Temperature-based $\mathrm{OCV}$}

The relationship between OCV and temperature has been discussed in Section II. Since the OCV will change with temperature, it is necessary to update the OCV when the temperature is changed. The calculation method is shown in (8). Note that the problem in [6] is that it only considered the temperature influence on resistance, but ignored the temperature influence on OCV.

$$
\frac{\partial U_{\text {term,time }(I)}}{\partial T}=U_{o c v(S o C, T)}-I \cdot \frac{\partial R(T)}{\partial T}
$$

\section{Temperature-based resistance}

According to (8), the terminal voltage could be measured and the change of OCV under different temperature conditions has been considered. Therefore, a resistance and temperature relationship function is generated.

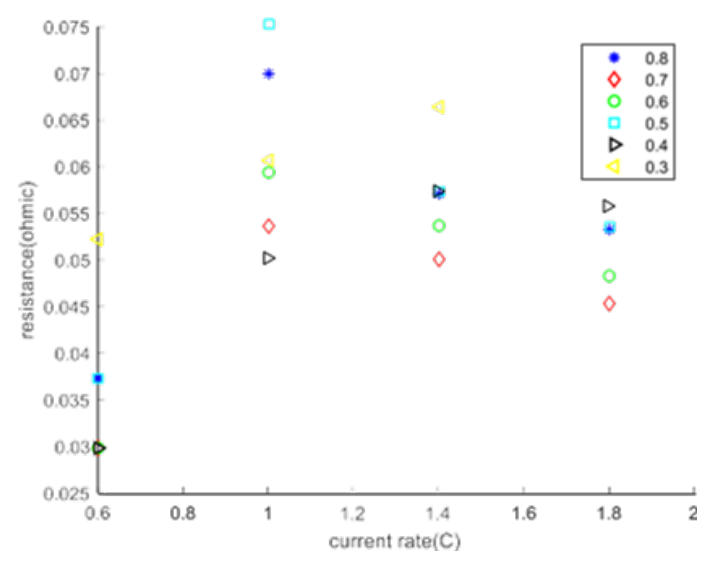

Fig. 4. Resistance-Current rate relationship.

\section{SoC-based $O C V$}

The SoC-based OCV curve has been generated by small current $(\mathrm{C} / 20)$ experiments. And the experimental results could be used to update resistance model.

\section{E. SoC-based resistance}

Choosing $1 \mathrm{C}$ rate as reference, the SoC-based model could be written as (9).

$$
R_{s o c}=\left(U_{o c v(s o c)}-U_{\text {term }(s o c, I)}\right) / I
$$

\section{RESULTS AND DISCUSSION}

\section{A. Data analysis}

The internal resistance data shown in Fig. 4 is calculated by (4) using different discharging current rates for $6 \mathrm{SoC}$ point. It can be found that for most SoC points, the internal resistance become smaller with the increase of current rate after 1C. For example, the maximum resistance value of $\mathrm{SoC}=0.5$ is 0.075 at $1.0 \mathrm{C}$. And it decreases to 0.057 and 0.053 at $1.4 \mathrm{C}$ and $1.8 \mathrm{C}$.

\section{B. Results Comparison}

Because the resistance of data fitting method is a constant value with the change of SoC and current rate, Fig. 5 is plotted based on the change of temperature. We apply testing data of $1.4 \mathrm{C}$ and $1.8 \mathrm{C}$ to (4) and (7) to estimate the resistance at $1.6 \mathrm{C}$, and the calculated value has been shown in Fig. 6. To compare the accuracy of proposed method, we use conventional pulse testing method to measure the resistance at $6 \mathrm{SoC}$ points, and the result has been shown in Fig. 6. According to Fig. 6, the proposed method and traditional pulse testing method have the similar result which are close to datasheet value, but they have a stark difference with the result of data fitting method, as shown in Fig. 5.

But according to [1], the mean absolute error and root mean squared error show that the $\mathrm{R}$ value of data fitting method is accurate in this dynamic street test. Although it has a comparable large difference with other two methods, it still can show a good performance in specific condition. 


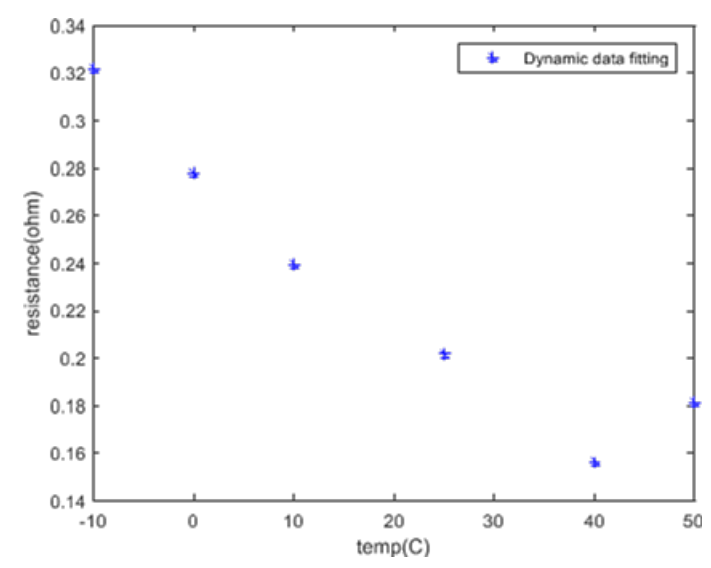

Fig. 5. Dynamic data fitting resistance at six temperature.

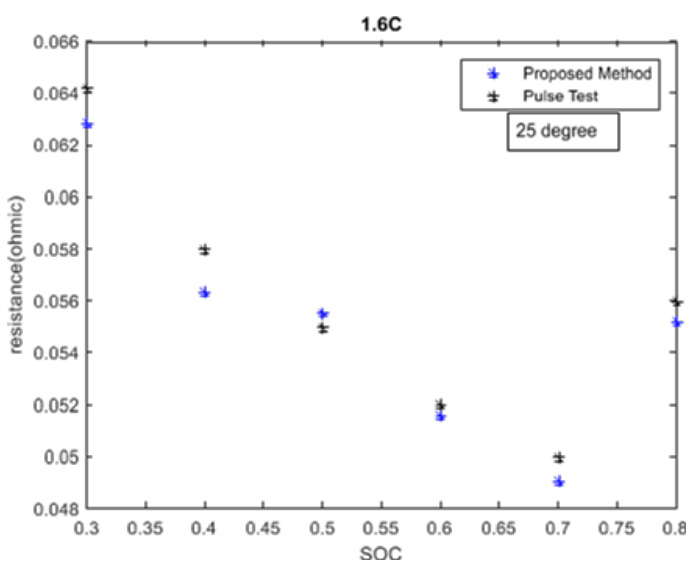

Fig. 6. The comparison result of pulse testing and proposed method.

With the increases in temperature and SoC, the internal resistance will decrease in a limited range. This could be verified by electrochemical aspect that the concentration polarization will decrease at medium to high temperature environment.

\section{CONCLUSION}

This paper proposed an improved internal resistance measurement method through combing the existing SoC- OCV data and dynamic street test data at six different ambient temperature, constant current charging test with four different current rates at standard ambient temperature condition $\left(25^{\circ} \mathrm{C}\right)$. And the proposed method has shown that the accuracy is higher than the data fitting method. The result shows that the proposed method has the similar value with traditional pulse testing, but the testing time is less than pulse testing which considers temperature and current rate factor.

\section{ACKNOWLEDGMENT}

This research was funded partially by the Australian Government through the Australian Research Council (Discovery Projects DP180100129).

\section{REFERENCES}

[1] Y. Xing, W. He, M. Pecht, and K. L. Tsui, "State of charge estimation of lithium-ion batteries using the open-circuit voltage at various ambient temperatures," Applied Energy., vol. 113, pp. 106-115, 2014.

[2] K.-T. Lee, M.-J. Dai, and C.-C. Chuang, "Temperature-compensated model for lithium-ion polymer batteries with extended kalman filter stateof-charge estimation for an implantable charger," IEEE Transactions on Industrial Electronics., vol. 65, no. 1, pp. 589-596, 2018.

[3] M. Coleman, W. G. Hurley, and C. K. Lee, "An improved battery characterization method using a two-pulse load test," IEEE Transactions on energy conversion., vol. 23, no. 2, pp. 708-713, 2008.

[4] L. Zheng, J. Zhu, G. Wang, D. D.-C. Lu, P. McLean, and T. He, "Experimental analysis and modeling of temperature dependence of lithiumion battery direct current resistance for power capability prediction," in Electrical Machines and Systems (ICEMS)., 2017 20th International Conference on, 2017, pp. 1-4: IEEE.

[5] F. Zheng, Y. Xing, J. Jiang, B. Sun, J. Kim, and M. Pecht, "Influence of different open circuit voltage tests on state of charge online estimation for lithium-ion batteries," Applied energy., vol. 183, pp. 513-525, 2016.

[6] Y. Chen, X. Liu, G. Yang, and H. Geng, "An internal resistance estimation method of lithium-ion batteries with constant current tests considering thermal effect," in Industrial Electronics Society., IECON 2017-43rd Annual Conference of the IEEE, 2017, pp. 7629-7634: IEEE.

[7] W. He, N. Williard, C. Chen, and M. Pecht, "State of charge estimation for Li-ion batteries using neural network modeling and unscented Kalman filter-based error cancellation," International Journal of Electrical Power and Energy Systems, vol. 62, pp. 783-791, 2014. 